\title{
Information-Based Intelligent Traffic Platform Design for City Parking Space Management
}

\author{
Zhang Xin \\ School Of Art And Design \\ Guangdong University of Technology \\ Guangzhou,China \\ uusmile@163.com
}

Keywords: Intelligent traffic; Information technology; Internet of Things; design

Abstract. With the development of wireless communication and Internet technologies, it is possible to see every parking stall as an information source. The Internet of Things technology can then facilitate the realization of automatic charging of static traffic (on-road parking) and real-time monitoring of parking stalls. If this information-based intelligent parking network is applied, it will help to build an intelligent management platform, which can achieve optimum use of parking space to effectively relieve traffic pressure in cities.

\section{Introduction}

The number of private cars in China keeps increasing, manual charge and meter charge, are very problematic, and they can not meet the demand of increasingly developed urban traffic. Outdoor parking lots generally have a sign and human-operated charge services, but there are no uniform standards about their charge. Many temporary parking stalls are unmarked but still charged. There are no clear guiding signs to outdoor parking lots. If drivers are unfamiliar with the roads, it will be a great trouble for them to find their way to the parking lot. Even if they finally find the parking lot, there is no staff ready to guide. Also, design of parking signs are out of fashion, which will affect urban landscape. Meter-based charge requires that drivers should first find the right meter after parking and then insert their card into meter to start the charging service. When they leave their parking stall, they should once again insert their card for settlement. Some drivers always forget the second inserting, thus wasting much money.

\section{Organization of the Text}

\section{CPN-The "Internet of Things" in Intelligent Traffic}

With the rapid development of the Internet, new technologies and systems such as Internet of Things and cloud computing are constantly emerging. Internet of Things is another revolution in information industry after computer, the Internet and mobile communication network. At present the Internet of Things finds very wide application in the following four major fields: environmental monitoring, tracing of things, intelligent power network and intelligent traffic. Among them, the first has already been in practice; the second is suspended due to weak basis; the third one has received attention; and the fourth one has huge potential.

Now intelligent traffic has reached a critical stage of development. Early intelligent traffic solutions focus on expressway. One of its important measures is to develop a well-functioning expressway tool system so as to exercise information-based management of all expressways across China. Today the biggest traffic problem is heavy traffic in cities. At this stage when the speed of road construction lags behind the growth rate of the number of cars, the key solution to heavy traffic is management of vehicles. 
In the future, intelligent traffic will focus on traffic hot spots, with the aim of changing the management mode of parking stalls. Therefore, it is an imperative to build a parking stall- based signal system: a parking stall network. This network incorporates available electronic information technologies, taking every parking stall as an information source and connecting it to the network via wireless communication techniques, thus able to achieve centralized management of all the parking stalls in China.

The CPN we design is an intelligent parking application based on "parking stall monitoring system and parking stall monitoring sensor". Incorporating techniques of Internet of Things as well as wireless communication and digital technology, this network can realize automatic charging of basic static traffic (on-road parking) and real-time monitoring of parking stalls. Connected to the sensor is "City Static Traffic Information Intelligent Management Platform”, which is mainly applied to city parking management and proves to be an effective solution to city static traffic management. This platform is a good substitute for the out-of-fashion meter charging. By means of wireless communication technology, it realizes smart management of urban traffic, which covers traffic planning, traffic information gathering, processing and releasing (including third-party information services), and mobile payment (namely payment via financial card, mobile phone, and others).

The CPN program, by use of available technology and hardware, aims to realize real-time, efficient and human-friendly management of parking in roads under civic planning and in all kinds of parks in urban districts. It can provide not only basic information such as an accurate statistics of urban means of transport, but also accurate feedbacks to relevant government departments. Above all, in times of parking lot shortage, it can be used to manage available parking lot resources and relieve traffic pressure. This program requires parking stalls in roads under civic planning and in business districts to be installed with parking stall detectors. The "City Static Traffic Information Intelligent Management Platform" combines all the detectable stalls and forms a network. This network will be an important window to understand digital Chinese cities.

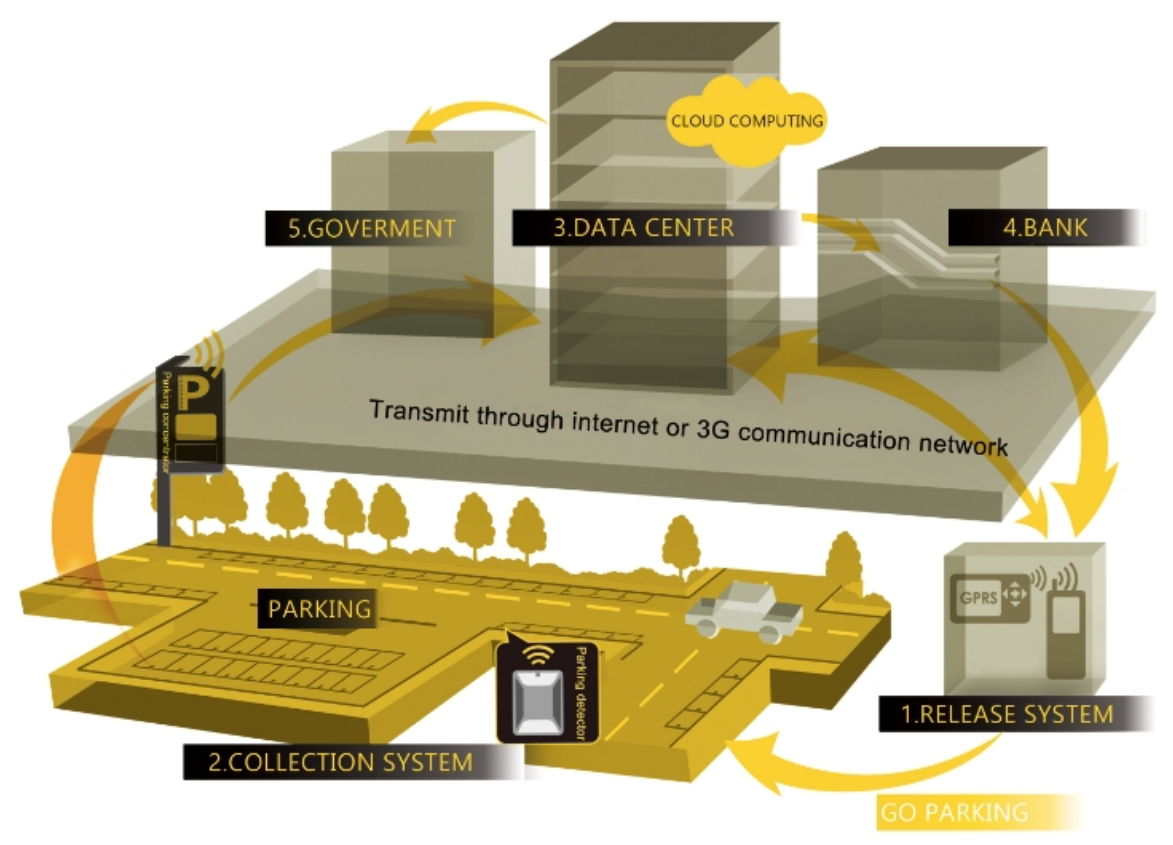

Fig 1. CPN-The "Internet of Things" in Intelligent Traffic 


\section{CPN User's Procedures}

(1) Check the parking stalls and toll mode of destination. Car owners can get information of a specific road by means of mobile phone applications, on-vehicle multimedia, webpages, etc. Relevant information includes the number of all parking stalls in this section of road, number of available parking stalls, parking toll mode and rate, whether parking is allowed in this section of road, and other sorts of information. Parking toll is either section-based, time-based, stall-based, parking times-based, or a combination of them.

(2) Register to be a user (for new users). Car owners can call the customer service center for registration, and if successful, the car owner will receive a new account sent by system. This account is bound with the mobile phone number of the owner, so in the future parking tolls would be deducted from the phone number account directly. At large parking lots, financial cards such as public traffic card and bank card can also be used.

(3) Charging. Car owners can buy rechargeable cards to do the account charging.

(4) Parking. Car owners can dial "Number + Parking Stall Number" to start timing of parking, and the owner will receive a reply message informing him/her the starting time of parking as well as the toll mode and rate. Timing of parking can also be done by a remote controller. When car owners want to park, all they need to do is to press the remote controller. Likewise, the parking stall monitoring system will send the owners a message informing them timing has started.

(5) Leaving. Car owners do not need to call again when leaving. When system stops timing, the parking time and toll will be summed up. All such information, together with the account balance, will be sent to users by messages. If non-users leave, a message will be sent to car owners immediately, so as to prevent burglars.

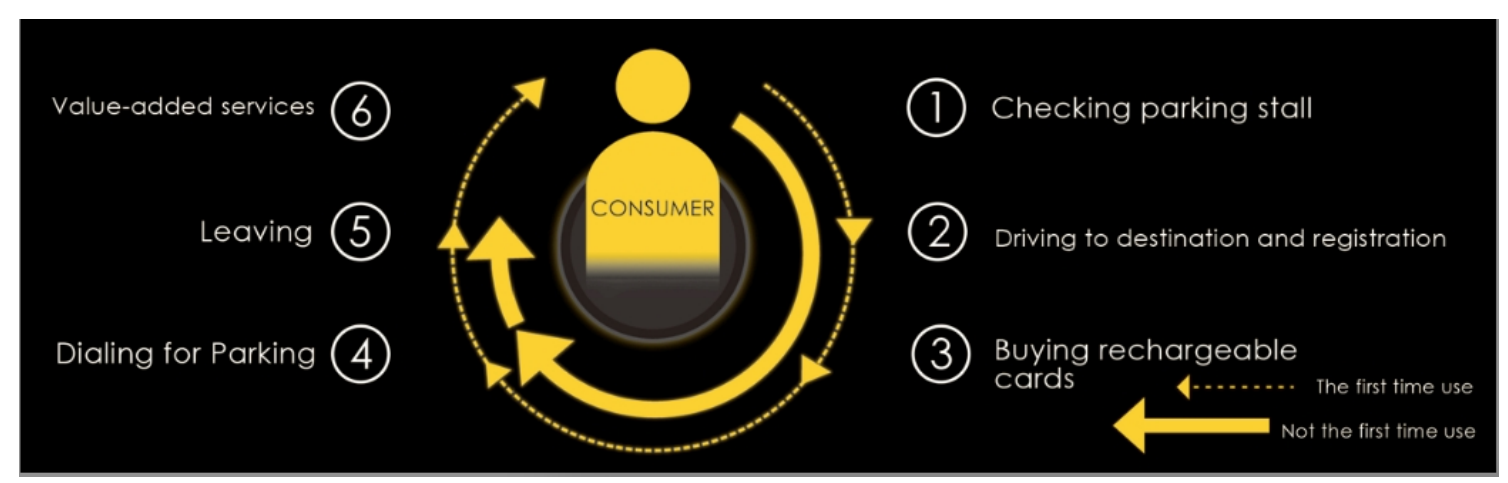

Fig 2. CPN User's Procedures

Left: 6. Value-added services; 5. Leaving; 4. Dialing for Parking

Middle: 1. Checking parking stall; 2. Driving to destination and registration; 3. Buying rechargeable cards Right: New U sers; N on-N ew U sers 


\section{CPN Product Designs}

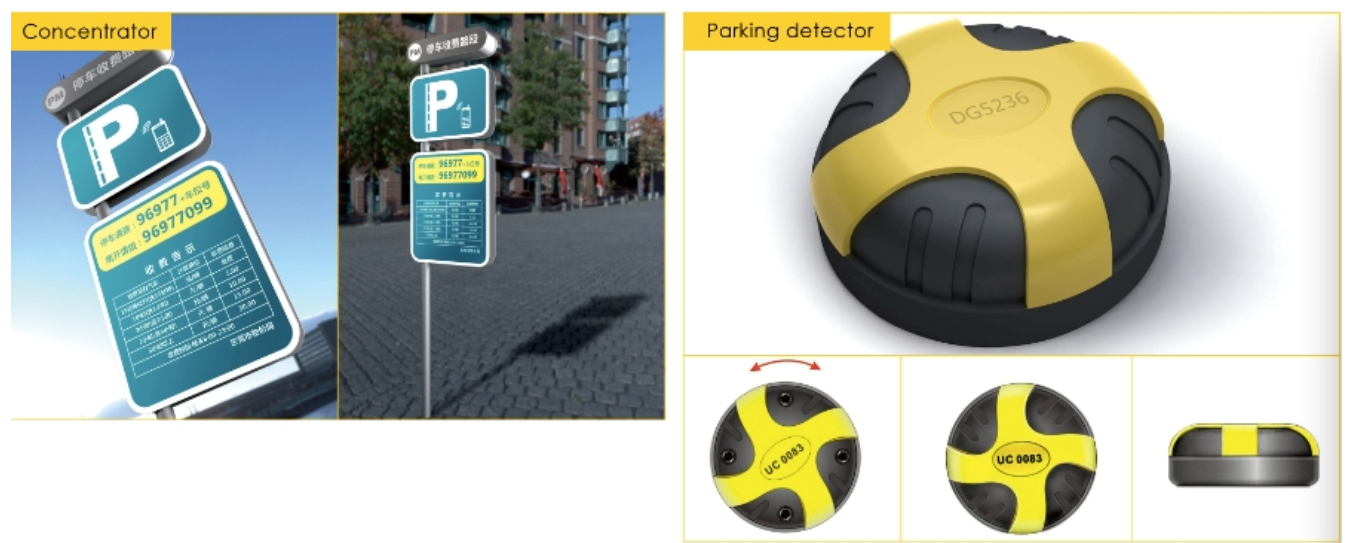

Fig 3. Drafts and Early Designs

(1) Fashion and simplicity. CPN, as its name suggests, is city-oriented. Parking space shortage and traffic jam are most serious in mega cities such as Beijing, Shanghai and Guangzhou. In the short run this program is applicable only to big cities, but later it will extend to second-tier cities. Therefore, the product designs in this program are targeted principally at mega cities. Designers seek to produce designs consistent with the orientation, environment, culture and aesthetic trends of these cities. After a large-scale analysis of many metropolises in and out of China, designers summarize the design style as "fashion and simplicity", which best represents the charm of modern metropolises.

(2) Differentiated analysis. Cities have different histories, cultures, environment and locations, which should never be neglected in product design. Designers should give full consideration to the characteristics of the city concerned, and incorporate elements of the city in the design to make the design more discriminating. Meanwhile, as product costs and corporate product style also matter, the designers also make small variations, like the design of head and choice of colors, to differentiate cities. The CPN design for Guangzhou is inspired by the Five Rams (a landmark statue in Guangzhou); and that for Dongguan is simply "DG", the acronym of the city name. 


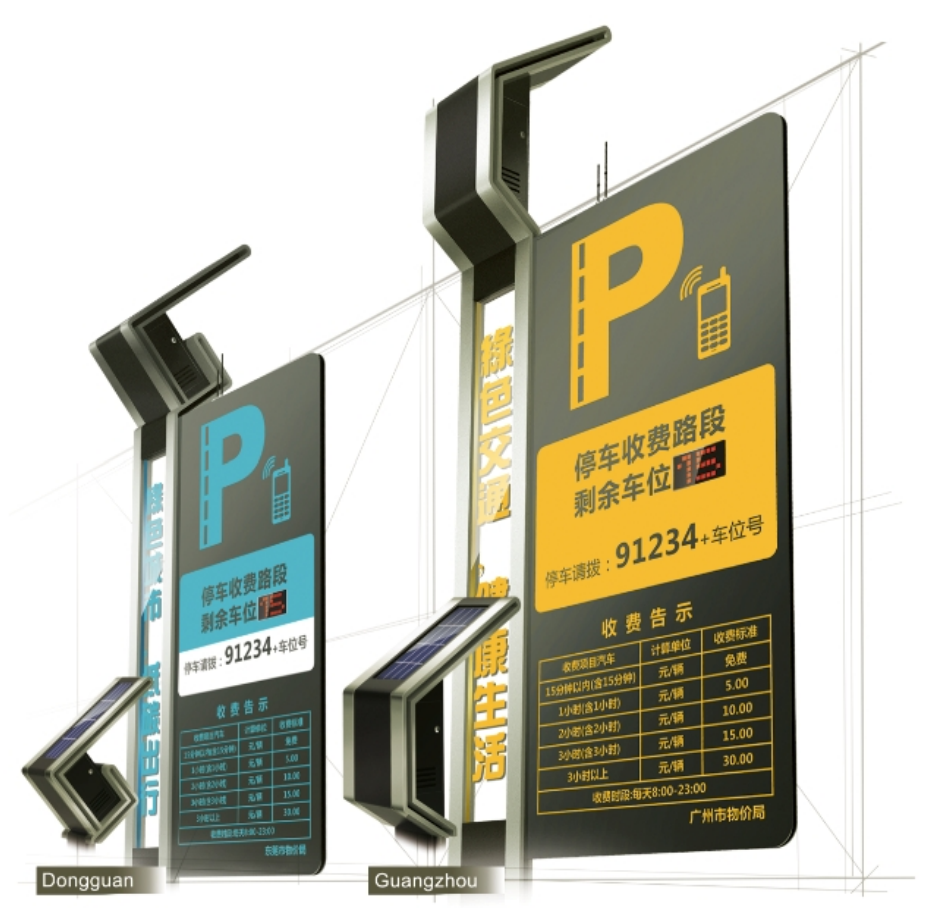

Fig 4. Differentiated Concentrator Designs in Guangzhou and Dongguan

(3) Environment-friendly. As CPN encourages "Green Traffic, Low-Carbon Life", its designs naturally take the issue of environmental protection into consideration. RF card is installed on both the parking stall concentrator and detector, so power supply is needed if they are to work. This is done by solar panels. Solar panels can not only guarantee working of equipment, but spares the trouble of changing batteries. Actually, it can ensure continuous power supply for ten days without sun. This is genuinely environment-friendly. In this program, low-energy consumption LED lights are used for night illumination of advertising slogans.

(4) Low cost, easy transportation and modular design. The concentrator is a modular design. It has three parts: head, body and billboard. Its head can be made either in the manner of sheet metal processing or mold opening, depending on the quantity needed. Its body and billboard are made customarily by mold opening. These parts can be assembled at site. All these design details can cut costs and make transportation easy. 


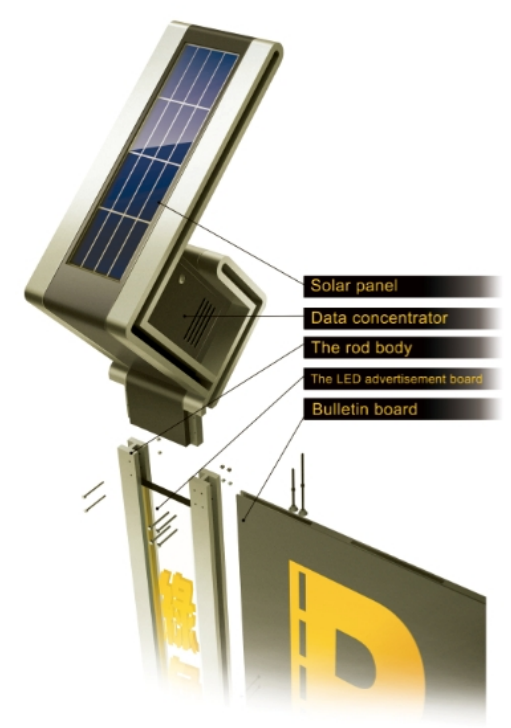

Fig 5. M odular Design of Data Concentrator

(5) Choice of colors. In this design, the billboard is either yellow and black or blue and white. From an ergonomic perspective, the color yellow has better visibility and thus is eye-catching, while black is the most recognizable color. That is why the two colors are often used together in the traffic management system. Blue and white are two colors with neutral color semantics, so in traffic management white words with blue background are often used together for signs and instructions. Therefore, this design also adopts these two color combinations, so that it follows the convention of the whole traffic system while conveying messages.
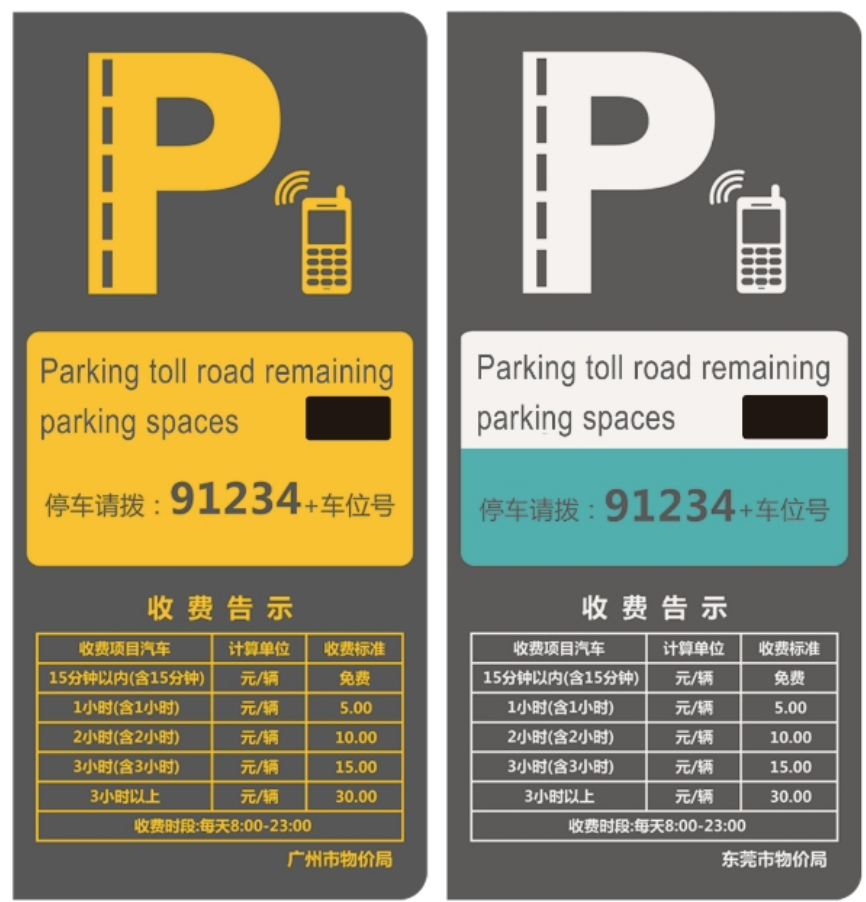

Fig 6. Concentrator Color Designs 

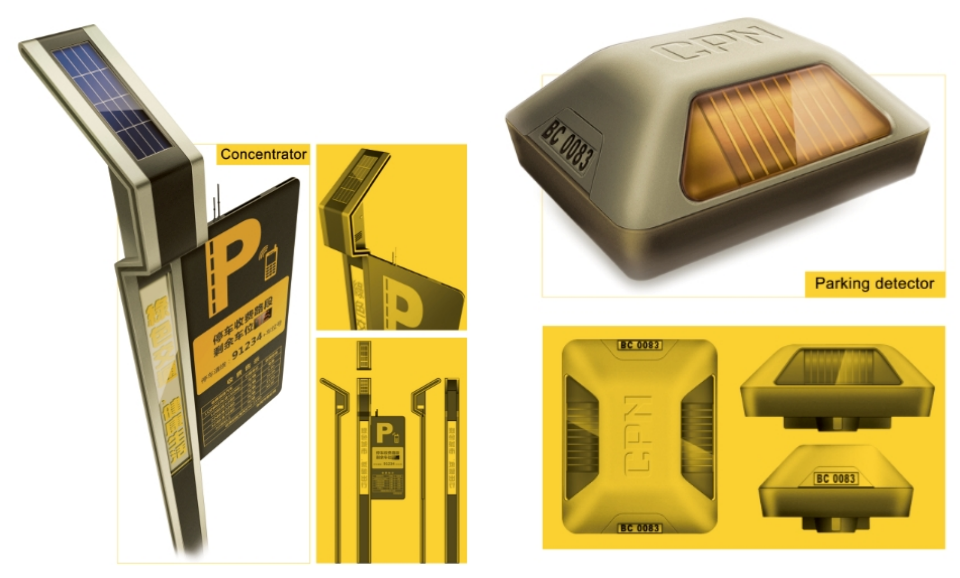

Fig 7. Design of Concentrator and Parking Stall M onitor

\section{Conclusion}

Now this design has been in commissioning in Dongguan, Haikou and Lin'an. After that, this design will be improved and promoted in other cities and even other fields. Governments will fund the CPN program; China Telecom or China Mobile will provide network services; and the program executing company will provide equipment and technical support. The CPN can realize real-time monitoring of the parking process of vehicles from their dynamic state to their static state. Car owners can get from it information about parking, navigation, vehicle emergency assistance, traffic maps, etc. Analysis of relevant data from the above three cities shows that the CPN can effectively alleviate traffic pressure and significantly improve service efficiency of roads. Number of traffic jams is reduced by $60 \%$; short-distance transport efficiency is improved by nearly $70 \%$; and traffic capacity of existing roads increases by 2 or 3 times. For cars with CPN services, its parking frequency decreases by 30\%; its working time decreases by $13 \%$ to $45 \%$; and its service efficiency improves by over $50 \%$. The CPN program realizes comprehensive, intelligent and automatic traffic management, and has advanced the development of digitalized traffic management and digitalized city.

\section{References}

[1] Lowrey L H, Banet M J, Lightner B, et al. Internet-based vehicle-diagnostic system: U.S. Patent 6,611,740[P]. 2003-8-26.Reference to a book:

[2] Beckert R D, Moeller M M, Wong W S. Vehicle computer system with wireless internet connectivity: U.S. Patent 6,202,008[P]. 2001-3-13.

[3] Sheth D G, Singh S. Enhanced vehicle controls through information transfer via a wireless communication system: U.S. Patent 6,405,106[P]. 2002-6-11.

[4] Caicedo F. Real-time parking information management to reduce search time, vehicle displacement and emissions[J]. Transportation Research Part D: Transport and Environment, 2010, 15(4): 228-234.

[5] YANG Ji-fei. Internet of Things-Based Intelligent Parking Payment System: Design and Implementation. Shanghai: Fudan University, 2010.

[6] LIU Jin and GU Jia-qiang. The Present Situation of the Internet of Things in China and Its Development Strategies. Enterprise Economy, 2013, (04): 23-26. [in Chinese]

[7]XU Chao, WANG Xinhong, and LIU Fuqiang. Internet of Vehicles: Architecture and Multichannel MAC. ZTE COMMUNICATIONS, 2011, 17(3), 12-15. [in Chinese] 
[8]SUN Xiao-hong. Key Technology and Its Application of IoV. Communications Technology, 2013(4), 6-9. [in Chinese] 